\title{
Current states in SNS junction for arbitrary concentrations of nonmagnetic impurities
}

\author{
A.V.Svidzinsky, V.E.Sakhnyuk \\ Department of Theoretical and Mathematical Physics, \\ Lesya Ukrainka Volyn State University, \\ 13 Voli Avenue, 43000 Lutsk, Ukraine
}

Received April 18, 2000

The boundary conditions are obtained for NS interface at the presence of arbitrary concentrations of nonmagnetic impurities. The current states in SNS junction are considered. The solution obtained for the current is analysed, in particular it was found out how the characteristic length of the current decreasing $\xi_{0}$ for pure superconductor turns to $\xi_{\mathrm{d}}$ for dirty one. A variant of the variational method, called the method of quasiorthogonality to asymptotics, is used to obtain the boundary conditions.

Key words: superconductor, order parameter, impurities, Green function

PACS: $74.50 .+r$

\section{Introduction}

To describe the current states in the superconducting junctions like SNS (superconductor - normal metal - superconductor) at the temperature not too close to the critical the model with a constant modulus of the order parameter is used. Here the availability of the coherent phase difference is assumed. It makes possible to describe the current states. In reality the order parameter modulus decreases near the border, but this change has a characteristic length $\xi_{0}$ - the coherence length and it effects only the numerical coefficient in the current for wide junctions, when the thickness $d$ is much larger than $\xi_{0}$. The simplified model cannot be applied at a temperature close to the critical, where the coherence length increases $\left(1-T / T_{\mathrm{c}}\right)^{-1 / 2}$ times. Therefore in this region of temperature one has to take into account spatial distribution of the order parameter. In this work we are interested in the current states at the temperature close to the critical. Both pure junction and the junction with nonmagnetic impurities are considered. It's natural to expect that the magnitude of such currents decreases, when $d$ increases. One can raise a question: what is a characteristic length of this decreasing? Since the order parameter close to the 
critical temperature is small, physical quantities of the linear by $\Delta$ approximation will be expressed by the Green function of the normal metal. This Green function has the characteristic length $\xi_{N}(T)$ of the order $v_{0} / T$; because $T \sim T_{\mathrm{c}}$, the characteristic length is $v_{0} / T$, i. e. it is of the order $\xi_{0}$. If the free path length is finite, then the characteristic length of the normal metal Green function equals $\left(1 / \xi_{0}+1 / l\right)^{-1}$. In other words, the characteristic length must be equal to $\min \left(\xi_{0}, l\right)$. Hence, just this characteristic length will determine the current decreasing when $d$ increases. In reality, as it's well known [1], if $l \ll \xi_{0}$, then $\xi_{\mathrm{d}}=\sqrt{\xi_{0} l / 3}$ will be the characteristic length. Thus the effect is associated not with a harmonic mean of $\xi_{0}$ and $l$, but with their geometric one. It's also known, that for $l \gg \xi_{0}, \xi_{0}$ becomes the characteristic length. Obviously, the appearance of new characteristic length is associated with the induction of weak superconductivity in the normal layer, but it's less obvious which way the smooth transition from $\sqrt{\xi_{0} l / 3}$ to $\xi_{0}$ takes place when the free path length increases. This question will be considered in the first part of the work. Another goal of the work is to build a new form of variation method that is more efficacious than Rits method and Gal'orkin method. This will be done in the second part of the work. Let's recall the scheme of building the superconductivity theory equations for the temperatures close to the critical. Since in this region of temperatures the order parameter is small, the set of the Gor'kov's equations can be solved by expanding the Gor'kov's Green functions by the degrees of the order parameter. As a result, we'll obtain the integral equation for the order parameter. Thus we shall obtain the integral equation for the order parameter. We assume additionally, that the order parameter changes slowly, then the obtained equation can be turned into the differential. Here the linear by $\Delta(\vec{r})$ terms will vanish and the expansion must be continued to cubic terms, which may be taken in a local form by $\Delta(\vec{r})$. The obtained nonlinear differential equation is the Ginzburg-Landau equation. So for the order parameter near the border we have the linear integral equation and at the distances much larger than the coherence length we obtain a nonlinear differential equation. We know its solution if the external magnetic field is absent. The Ginzburg-Landau equation is just for the distances from the border of the order $\xi_{0}$. It can be used to obtain the boundary condition for the Ginzburg-Landau equation. Such a possibility arises because there exists a spatial region where both the linear integral equation and the Ginzburg-Landau equation are true. For solutions of the integral equation this is the region of the large distances from the border (comparing to $\xi(T))$. Asymptotics of the linear integral equation proves to be the linear function. It's important, that the relation of two coefficients of this function is determined from the equation. In case of SN interface it will be denoted as $q_{\infty}$ and it has to be calculated explicitly. The parameter $q_{\infty}$ determines a boundary condition for the Ginzburg-Landau equation - the tangent of a slope angle of the dimensionless order parameter to the abscissa axis in zero. For SNS junction two linear equations arise, their asymptotics being the linear functions as well, and such two parameters take place. Since the current is expressed by these parameters they should be calculated as well. 


\section{SNS junction in the presence of impurities}

It's known that the set of the linear integral equations, that arise for the order parameter of a superconductor in the presence of impurities, has the form

$$
\begin{aligned}
\Delta(z) & =\frac{\pi \rho T_{\mathrm{c}}}{v_{0}} \sum_{\omega_{n}} \int_{0}^{1} \frac{\mathrm{d} x}{x} \int_{-\infty}^{\infty} \exp \left(-\frac{2\left|\omega_{n}^{\prime}\right|}{v_{0}}\left|z-z^{\prime}\right|\right) \Delta_{\omega_{n}}\left(z^{\prime}\right) \mathrm{d} z^{\prime}, \\
\Delta_{\omega_{n}}(z) & =\Delta(z)+\frac{1}{2 l} \int_{0}^{1} \frac{\mathrm{d} x}{x} \int_{-\infty}^{\infty} \exp \left(-\frac{2\left|\omega_{n}^{\prime}\right|}{v_{0}}\left|z-z^{\prime}\right|\right) \Delta_{\omega_{n}}\left(z^{\prime}\right) \mathrm{d} z^{\prime} .
\end{aligned}
$$

Here $\left|\omega_{n}^{\prime}\right|=\left|\omega_{n}\right|+1 / \tau, \quad \omega_{n}=\pi T_{\mathrm{c}}(2 n+1)$ - Matsubara's frequency, $\tau$ - mean free time; $\tau=l / v_{0}, v_{0}$ - Fermi-velocity; $\rho=|g| N(0)$ - dimensionless attraction constant, $N(0)$ - electron state density on the surface of the Fermi-sphere. Let the surfaces, that separate normal and superconductive metals, be planar and the axis $O Z$ be perpendicular to them. In such a geometry the spatial homogeneity is broken in the direction of the axis $O Z$ that is clear from the equations (1) and (2). To simplify our dealing with equations (1) and (2) it's convenient to write them in the dimensionless form. Supposing $z \equiv \zeta \xi_{0}$ we obtain

$$
\begin{aligned}
\Delta_{n}(\zeta) & =\Delta(\zeta)+\frac{1}{2 \lambda} \int_{0}^{1} \frac{\mathrm{d} x}{x} \int_{-\infty}^{\infty} \exp \left(-\frac{\left|\zeta-\zeta^{\prime}\right|}{x}\left|2 n^{\prime}+1\right|\right) \Delta_{n}\left(\zeta^{\prime}\right) \mathrm{d} \zeta^{\prime}, \\
\Delta(\zeta) & =\frac{\rho}{2} \sum_{n} \int_{0}^{1} \frac{\mathrm{d} x}{x} \int_{-\infty}^{\infty} \exp \left(-\frac{\left|\zeta-\zeta^{\prime}\right|}{x}\left|2 n^{\prime}+1\right|\right) \Delta_{n}\left(\zeta^{\prime}\right) \mathrm{d} \zeta^{\prime} .
\end{aligned}
$$

Here $\left|2 n^{\prime}+1\right|=|2 n+1|+1 / \lambda, \quad \lambda=l / \xi_{0}$ - dimensionless electron free path.

Equation (3) can be easily solved by Fourier transformation relative to $\tilde{\Delta}_{n}(k)$. Using this solution we can exclude $\Delta_{n}$ and write a closed linear integral equation for the order parameter

$$
\Delta(\zeta)=\int_{-\infty}^{\infty} K\left(\zeta-\zeta^{\prime}\right) \Delta\left(\zeta^{\prime}\right) \mathrm{d} \zeta^{\prime}
$$

Fourier-component of the kernel of this equation has the form

$$
\tilde{K}(k)=\rho \sum_{n}\left[\frac{1}{k} \arctan \frac{k}{\left|2 n^{\prime}+1\right|}\right] /\left[1-\frac{1}{\lambda k} \arctan \frac{k}{\left|2 n^{\prime}+1\right|}\right] .
$$

Using variational methods for equation (5) one can obtain the boundary condition for the Ginzburg-Landau equation in the case of NS boundary. Omitting this (see $[1, \S 40])$ we shall investigate the current states in SNS junction. The linear integral equation (5) for the order parameter in the case of SNS junction has the form

$$
\Delta(\zeta)=\int_{-\infty}^{-a / 2} K\left(\zeta-\zeta^{\prime}\right) \Delta\left(\zeta^{\prime}\right) \mathrm{d} \zeta^{\prime}+\int_{a / 2}^{\infty} K\left(\zeta-\zeta^{\prime}\right) \Delta\left(\zeta^{\prime}\right) \mathrm{d} \zeta^{\prime}
$$


because $\Delta$ vanishes in a normal layer, i. e. in the region $|z|<d / 2$, or $|\zeta|<a / 2$, $a=d / \xi_{0}$. The equation can be reduced to the equation on the half-axis $\zeta>0$ if we introduce the even $\Delta_{\mathrm{s}}(\zeta)$ and the odd $\Delta_{\mathrm{a}}(\zeta)$ parts of the parameter $\Delta(\zeta)$ and carry out the shift on $a / 2$. After the simple transformation we obtain (shifted functions are denoted by the same letters)

$$
\Delta_{s, a}(\zeta)=\int_{0}^{\infty}\left\{K\left(\zeta-\zeta^{\prime}\right) \pm K\left(\zeta+\zeta^{\prime}+a\right)\right\} \Delta_{\mathrm{s}, \mathrm{a}}\left(\zeta^{\prime}\right) \mathrm{d} \zeta^{\prime}
$$

Sign $(+)$ corresponds to the even part of the function $\Delta(\zeta)$ and $(-)$ - to the odd part of it. The explicit form of the kernel $K\left(\zeta-\zeta^{\prime}\right)$ in the configuration space can be obtained in the limit cases $\lambda \sim 0$ and $\lambda \gg 1$, i. e. in the cases of pure and dirty superconductors. We shall consider the common scheme of description for whole the interval of lengths $\lambda$.

Proceeding to the solution of equation (8), let us separate the asymptotics on the infinity

$$
\Delta(\zeta)=\zeta+q_{\infty}+\psi(\zeta)
$$

We still do not put indices $\mathrm{s}$ and a near $\Delta(\zeta)$ and $q_{\infty}$, because of the likeness of the equation for them which is enough to consider the equation only for one of the functions, for example, for $\Delta_{s}(\zeta)$. After the substitution shown we obtain

$$
\begin{aligned}
\psi(\zeta) & -\int_{0}^{\infty} K\left(\zeta-\zeta^{\prime}\right) \psi\left(\zeta^{\prime}\right) \mathrm{d} \zeta^{\prime}= \\
& =\int_{0}^{\infty} K\left(\zeta+\zeta^{\prime}\right) \zeta^{\prime} \mathrm{d} \zeta^{\prime}-q_{\infty} \int_{0}^{\infty} K\left(\zeta+\zeta^{\prime}\right) \mathrm{d} \zeta^{\prime}+\int_{0}^{\infty} K\left(\zeta+\zeta^{\prime}+a\right) \Delta\left(\zeta^{\prime}\right) \mathrm{d} \zeta^{\prime}
\end{aligned}
$$

We shall find the solution using the perturbation theory, taking into account only the first order correction

$$
\psi(\zeta)=\psi^{(0)}(\zeta)+\psi^{(1)}(\zeta), \quad q_{\infty}=q_{\infty}^{(0)}+\delta q_{\infty}
$$

The equation for the zero approximation has the form

$$
\psi^{(0)}(\zeta)-\int_{0}^{\infty} K\left(\zeta-\zeta^{\prime}\right) \psi^{(0)}\left(\zeta^{\prime}\right) \mathrm{d} \zeta^{\prime}=\int_{0}^{\infty} K\left(\zeta+\zeta^{\prime}\right) \zeta^{\prime} \mathrm{d} \zeta^{\prime}-q_{\infty}^{(0)} \int_{0}^{\infty} K\left(\zeta+\zeta^{\prime}\right) \mathrm{d} \zeta^{\prime}
$$

In the first approximation

$$
\begin{aligned}
& \psi^{(1)}(\zeta)-\int_{0}^{\infty} K\left(\zeta-\zeta^{\prime}\right) \psi^{(1)}\left(\zeta^{\prime}\right) \mathrm{d} \zeta^{\prime}= \\
& =-\delta q_{\infty} \int_{0}^{\infty} K\left(\zeta+\zeta^{\prime}\right) \mathrm{d} \zeta^{\prime}+\int_{0}^{\infty} K\left(\zeta+\zeta^{\prime}+a\right) \Delta^{(0)}\left(\zeta^{\prime}\right) \mathrm{d} \zeta^{\prime}
\end{aligned}
$$


Solution of equation (9) can be found from the condition of orthogonality of the right side to the solution of the corresponding uniform equation, i. e. to $\Delta^{(0)}(\zeta)$. Thus, we have

$$
\delta q_{\infty}=\frac{1}{2 I_{1}} \int_{0}^{\infty} \mathrm{d} \zeta \Delta^{(0)}(\zeta) \int_{0}^{\infty} K\left(\zeta+\zeta^{\prime}+a\right) \Delta^{(0)}\left(\zeta^{\prime}\right) \mathrm{d} \zeta^{\prime} .
$$

Here the identity proved in [2] was taken into account:

$$
\int_{0}^{\infty} \mathrm{d} \zeta \Delta^{(0)}(\zeta) \int_{0}^{\infty} K\left(\zeta+\zeta^{\prime}\right) \mathrm{d} \zeta^{\prime}=2 I_{1}
$$

Let us use the solution of the equation of zero approximation obtained by the variational method: $\Delta^{(0)}(\zeta) \cong \zeta+\Gamma$, where $\Gamma=I_{1} / I_{0}$ [1]. Thus, for $\delta q_{\infty}$ one obtains

$$
\delta q_{\infty}=\frac{1}{2}\left(\frac{I_{2}(a)}{I_{1}}+2 \frac{I_{1}(a)}{I_{0}}+\frac{I_{1}}{I_{0}^{2}} I_{0}(a)\right)
$$

where

$$
\begin{aligned}
& I_{0}(a)=\int_{0}^{\infty} \mathrm{d} \zeta \int_{0}^{\infty} \mathrm{d} \zeta^{\prime} K\left(\zeta+\zeta^{\prime}+a\right), \\
& I_{1}(a)=\int_{0}^{\infty} \mathrm{d} \zeta \int_{0}^{\infty} \mathrm{d} \zeta^{\prime} \zeta^{\prime} K\left(\zeta+\zeta^{\prime}+a\right), \\
& I_{2}(a)=\int_{0}^{\infty} \mathrm{d} \zeta \zeta \int_{0}^{\infty} \mathrm{d} \zeta^{\prime} \zeta^{\prime} K\left(\zeta+\zeta^{\prime}+a\right) .
\end{aligned}
$$

An expression for the current is given by a common formula of the Ginzburg-Landau theory [2] (that should be calculated)

$$
j=\frac{6}{7 \zeta(3)} \frac{e n v_{0}}{p_{0} \xi_{0}}\left(1-\frac{T}{T_{\mathrm{c}}}\right)^{2} \delta q_{\infty} \sin \varphi
$$

This formula, however, contains $\delta q_{\infty}$, that should be found. Thus, describing the current states in SNS junction we shall deal with the expression for current (12), where $\delta q_{\infty}$ is represented by formula (10) with yet unknown magnitudes of the integrals $I_{1}(a), I_{2}(a), I_{3}(a)$. Since for the wide SNS junction $\left(d \gg \xi_{0}\right)$ the current decreases exponentionally if the normal metal layer's width increases, a characteristic length of such a decrease appears. It equals $\xi_{0}$ for the pure superconductor and $\xi_{\mathrm{d}}$ for the extremely dirty one. This dependence has the origin in the mentioned integrals. Thus, to investigate the transition from one characteristic length to another, one has to transform these integrals to a convenient form.

We shall carry out the calculation in detail for one of them, e. g. for $I_{0}(a)$, the scheme is the same for all of the three. 
Let us consider

$$
I_{0}(a)=\int_{-\infty}^{\infty} \mathrm{d} \zeta \int_{-\infty}^{\infty} \mathrm{d} \zeta^{\prime} K\left(\zeta+\zeta^{\prime}+a\right)
$$

First of all, let's express $K(\zeta)$ by Fourier component $\tilde{K}(k)$, an expression for the latter is given by equation (6). Let

$$
K(\zeta)=\frac{1}{2 \pi} \int_{-\infty}^{\infty} \tilde{K}(k) \mathrm{e}^{-\mathrm{i} k \zeta} \mathrm{d} k
$$

Then

$$
\begin{aligned}
I_{0}(a) & =\frac{1}{2 \pi} \int_{-\infty}^{\infty} \mathrm{d} k \tilde{K}(k) \mathrm{e}^{-\mathrm{i} k a} \int_{0}^{\infty} \mathrm{d} \zeta^{\prime} \int_{0}^{\infty} \mathrm{d} \zeta \mathrm{e}^{-\mathrm{i} k\left(\zeta+\zeta^{\prime}\right)} \\
& =-\frac{1}{2 \pi} \int_{-\infty}^{\infty} \frac{\tilde{K}(k) \mathrm{e}^{-\mathrm{i} k a}}{(k-\mathrm{i} \varepsilon)^{2}} \mathrm{~d} k, \quad \varepsilon \rightarrow 0 .
\end{aligned}
$$

In the same manner

$$
I_{1}(a)=\frac{\mathrm{i}}{2 \pi} \int_{-\infty}^{\infty} \frac{\tilde{K}(k) \mathrm{e}^{-\mathrm{i} k a}}{(k-\mathrm{i} \varepsilon)^{3}} \mathrm{~d} k, \quad I_{2}(a)=\frac{1}{2 \pi} \int_{-\infty}^{\infty} \frac{\tilde{K}(k) \mathrm{e}^{-\mathrm{i} k a}}{(k-\mathrm{i} \varepsilon)^{4}} \mathrm{~d} k, \quad \varepsilon \rightarrow 0 .
$$

Let us use the relation

$$
\frac{1}{(k-\mathrm{i} \varepsilon)^{n}}=\frac{P f}{k^{n}}+\mathrm{i} \pi \frac{(-1)^{n-1}}{(n-1) !} \delta^{(n-1)}(k),
$$

and integration rule of the pseudo function $P f / k^{n}$ with function $\varphi(k)$ :

$$
\int_{-\infty}^{\infty} \frac{P f}{k^{n}} \varphi(k) \mathrm{d} k=\int_{-\infty}^{\infty}\left[\varphi(k)-\varphi(0)-k \varphi^{\prime}(0)-\ldots-\frac{k^{n-2}}{(n-2) !} \varphi^{(n-2)}(0)\right] \frac{\mathrm{d} k}{k^{n}} .
$$

For the integral $I_{0}(a)$ we have

$$
\begin{aligned}
I_{0}(a) & =-\frac{1}{2 \pi} \int_{-\infty}^{\infty} \frac{\tilde{K}(k) \mathrm{e}^{-\mathrm{i} k a}-1}{k^{2}} \mathrm{~d} k-\frac{\mathrm{i}}{2} \lim _{k \rightarrow 0}\left(\tilde{K}(k) \mathrm{e}^{-\mathrm{i} k a}\right)^{\prime} \\
& =\frac{1}{2 \pi} \int_{-\infty}^{\infty} \frac{1-\tilde{K}(k) \cos k a}{k^{2}} \mathrm{~d} k-\frac{a}{2}=\frac{1}{2 \pi} \int_{-\infty}^{\infty} \frac{(1-\tilde{K}(k)) \cos k a}{k^{2}} \mathrm{~d} k .
\end{aligned}
$$

Here we used evenness of function $\tilde{K}(k)$, equality $\tilde{K}^{\prime}(0)=0$ and the elementary integral $\int_{-\infty}^{\infty}\left[(1-\cos k a) / k^{2}\right] \mathrm{d} k=\pi a$. The analogous expression for integrals $I_{1}(a)$ and $I_{2}(a)$ are given by representations

$$
\begin{aligned}
& I_{1}(a)=\frac{1}{2 \pi} \int_{-\infty}^{\infty}\left(\tilde{K}(k)-1-\frac{1}{2} k^{2} \tilde{K}^{\prime \prime}(0)\right) \sin k a \frac{\mathrm{d} k}{k^{3}} \\
& I_{2}(a)=\frac{1}{2 \pi} \int_{-\infty}^{\infty}\left(\tilde{K}(k)-1-\frac{1}{2} k^{2} \tilde{K}^{\prime \prime}(0)\right) \cos k a \frac{\mathrm{d} k}{k^{4}}
\end{aligned}
$$


To calculate $\tilde{K}^{\prime \prime}(0)$ we use an asymptotical expression for a small $k$

$$
\tilde{K}(k)-1 \stackrel{\text { as }}{=} \frac{\rho}{3} \sum_{n} \frac{k^{2}}{(2 n+1)^{2}\left|2 n^{\prime}+1\right|}, \quad k \sim 0 .
$$

From here

$$
\tilde{K}^{\prime \prime}(0)=\frac{2}{3} \rho \sum_{n} \frac{1}{(2 n+1)^{2}\left|2 n^{\prime}+1\right|} .
$$

Let us replace the kernel $\tilde{K}(k)$ by its implicit expression and change the variable in the integrals $I_{0}(a), I_{1}(a), I_{2}(a)$ so that $k=\left|2 n^{\prime}+1\right| x$. Taking into account, that $1=\rho \sum_{n} 1 /|2 n+1|$, we obtain

$$
\begin{aligned}
& I_{0}(a)=\frac{\rho}{\pi} \int_{-\infty}^{\infty} \frac{x-\arctan x}{1+(x-\arctan x) / \lambda x} \cos \left[\left(1+\frac{1}{\lambda}\right) a x\right] \frac{\mathrm{d} x}{x^{3}} \\
& I_{1}(a)=\frac{\rho}{\pi} \frac{\lambda}{1+\lambda} \int_{-\infty}^{\infty}\left(\frac{\arctan x-x}{1+(x-\arctan x) / \lambda x}+\frac{x^{3}}{3}\right) \sin \left[\left(1+\frac{1}{\lambda}\right) a x\right] \frac{\mathrm{d} x}{x^{4}} \\
& I_{2}(a)=\frac{\rho}{\pi} \frac{\lambda^{2}}{(1+\lambda)^{2}} \int_{-\infty}^{\infty}\left(\frac{\arctan x-x}{1+(x-\arctan x) / \lambda x}+\frac{x^{3}}{3}\right) \cos \left[\left(1+\frac{1}{\lambda}\right) a x\right] \frac{\mathrm{d} x}{x^{5}} .
\end{aligned}
$$

Here we used the fact, that for $d \gg \xi_{0}$ the main contribution to the sum is given by $n=0$ and $n=-1$. For the next consideration of these integrals we proceed to the complex variables and use the logarithmic representation of $\operatorname{arctangent} \operatorname{Arctan} z=$ $\operatorname{Ln}[(1+\mathrm{i} z) /(1-\mathrm{i} z)] / 2 \mathrm{i}$.

Let us integrate by contour closed in the upper half-plane, a cut being done by an imaginary axis and the point of branching $z=\mathrm{i}$ being by-passed as it's shown in figure 1.

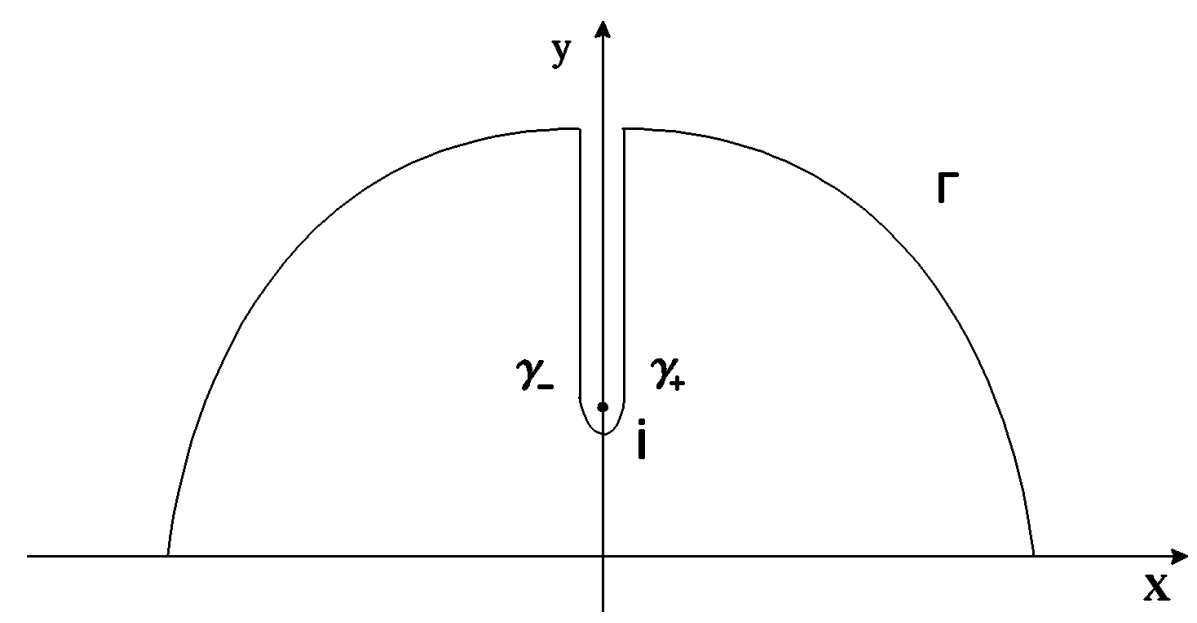

Figure 1. Path of integration for estimation of the integrals $I_{s}(a)$.

The contour contains a single pole, which is determined by the condition of equality of the function $1+1 / \lambda-1 / \lambda z \arctan z$ to zero. The pole lies on the imaginary 
axis $\left(z=\mathrm{i} y_{0}, y_{0}<1\right)$. When integration by the imaginary axis we define a logarithm so that on the left side $\left(\gamma_{+}\right) \operatorname{Ln}(1-y)=\ln (y-1)+\mathrm{i} \pi$ and on the right side $\left(\gamma_{-}\right)$ $\operatorname{Ln}(1-y)=\ln (y-1)-\mathrm{i} \pi$.

Using the residue theorem we obtain

$$
\begin{aligned}
I_{s}(a)= & \rho \frac{\lambda^{s+2}}{(1+\lambda)^{s}} \int_{1}^{\infty} \frac{\exp \{-a(1+1 / \lambda) y\}}{(1+\lambda+\ln [(y-1) /(y+1)] / 2 y)^{2}+\pi^{2} / 4 y^{2}} \frac{\mathrm{d} y}{y^{3+s}} \\
& +\rho \frac{\lambda^{s+2}}{(1+\lambda)^{s}} \frac{2\left(1-y_{0}^{2}\right)}{y_{0}^{1+s}} \frac{\exp \left\{-a(1+1 / \lambda) y_{0}\right\}}{(1+\lambda) y_{0}^{2}-\lambda}, \quad s=0,1,2 .
\end{aligned}
$$

From this formula one can see that the integrals and, consequently, the magnitude of the current through the junction decrease exponentially. To understand how the characteristic length of decreasing is formed, let us consider both addends in (20). In the pole one we have an exponent

$$
\exp \left\{-a\left(1+\frac{1}{\lambda}\right) y_{0}\right\}=\exp \left\{-d\left(\frac{1}{\xi_{0}}+\frac{1}{l}\right) y_{0}\left(\frac{l}{\xi_{0}}\right)\right\}
$$

from where we see that the magnitude of the pole has an effect upon the forming of the characteristic length. The root of the corresponding equation depends on the concentration of impurities and can be found numerically (see figure 2).

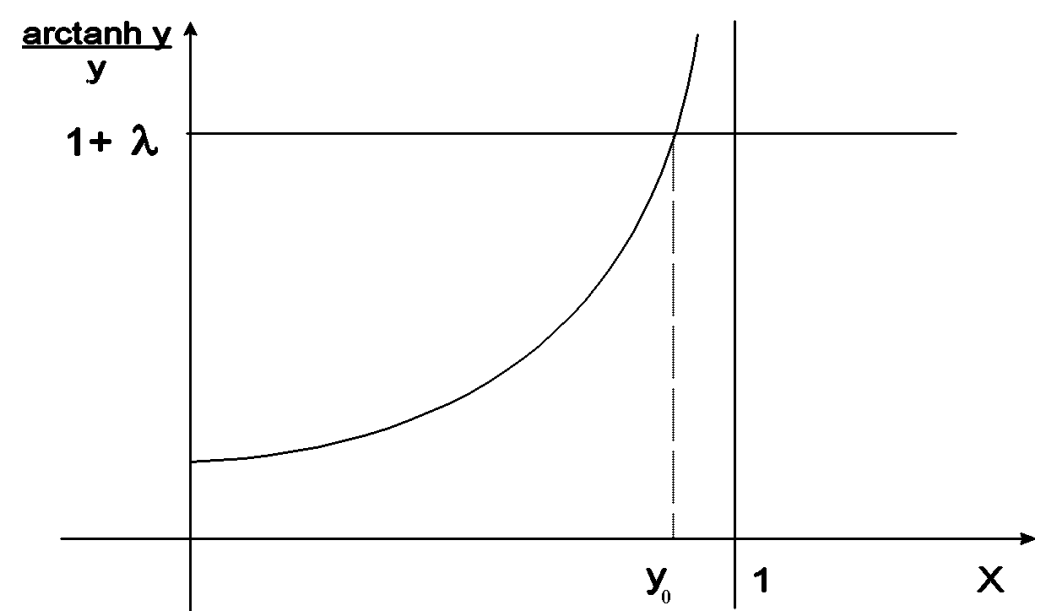

Figure 2. Pole of integrand in the integrals $I_{s}(a)$.

As for the integral addend, it can be evaluated by Laplace method, and the expression that determines the dependence in $d$ can be expressed in the form

$$
\exp \left\{-d\left(\frac{1}{\xi_{0}}+\frac{1}{l}\right)\right\} y_{1}\left(\frac{l}{\xi_{0}}\right) \text {. }
$$

Here $y_{1}\left(l / \xi_{0}\right)$ should be found from the condition of the minimum of the function

$$
a\left(1+\frac{1}{\lambda}\right) y+\ln \left\{\left(1+\frac{1}{\lambda}+\frac{1}{2 y \lambda} \ln \frac{y-1}{y+1}\right)^{2}+\frac{\pi^{2}}{4 y^{2} \lambda^{2}}\right\}
$$


i.e. from equation

$$
\begin{aligned}
a+\frac{a}{\lambda}= & \frac{1}{\lambda}\left[\left(1+\frac{1}{\lambda}+\frac{1}{2 y_{1} \lambda} \ln \frac{y_{1}-1}{y_{1}+1}\right)\left(\frac{1}{y_{1}^{2}} \ln \frac{y_{1}-1}{y_{1}+1}-\frac{2}{y_{1}} \frac{1}{y_{1}^{2}-1}\right)+\frac{\pi^{2}}{2 y_{1}^{3} \lambda_{1}}\right] \\
\times & {\left[\left(1+\frac{1}{\lambda}+\frac{1}{2 y_{1} \lambda} \ln \frac{y_{1}-1}{y_{1}+1}\right)^{2}+\frac{\pi^{2}}{4 y_{1}^{2} \lambda^{2}}\right]^{-1} }
\end{aligned}
$$

Thus the characteristic length of the current decreasing is inversed to the expression $\left(1 / \xi_{0}+1 / l\right) f\left(l / \xi_{0}\right)$, where $f\left(l / \xi_{0}\right)$ is either $y_{0}\left(l / \xi_{0}\right)$ or $y_{1}\left(l / \xi_{0}\right)$.

Let us consider the extreme cases $\lambda \sim 0$ and $\lambda \gg 1$. If $\lambda \sim 0$ then the main contribution to (20) is made by the pole. Asymptotics of the contribution has the form

$$
\begin{aligned}
& 2 \rho \frac{\lambda^{2+s}}{(1+\lambda)^{s}} \frac{1-y_{0}^{2}}{y_{0}^{1+s}} \frac{\exp \left\{-a(1+1 / \lambda) y_{0}\right\}}{(1+\lambda) y_{0}^{2}-\lambda} \stackrel{\text { as }}{=} \\
& \quad \stackrel{\text { as }}{=} 2 \rho \frac{\lambda^{2+s}}{y_{0}^{1+s}} \frac{\exp \left\{-\frac{a}{\lambda} y_{0}\right\}}{y_{0}^{2}-\lambda}=\rho\left(\frac{\lambda}{3}\right)^{\frac{s+1}{2}} \exp \left\{-a \sqrt{\frac{3}{\lambda}}\right\} .
\end{aligned}
$$

From this for the integrals $I_{s}(a), s=0,1,2$, we have

$$
I_{s}(a) \cong \rho\left(\frac{\lambda}{3}\right)^{\frac{s+1}{2}} \exp \left(-a \sqrt{\frac{3}{\lambda}}\right), s=0,1,2, \quad \lambda \ll 1 .
$$

And accordingly, for the current we obtain

$$
j \cong 0,5 \frac{e n v_{0}}{p_{0} \xi_{0}} \sqrt{\frac{l}{\xi_{0}}} \mathrm{e}^{-d \sqrt{3 / \xi_{0} l}}\left(1-\frac{T}{T_{\mathrm{c}}}\right)^{2} \sin \varphi, d \gg \sqrt{\frac{\xi_{0} l}{3}} .
$$

In the limit $\lambda \gg 1$ the main contribution to (20) is made by the integral term, that asymptotically for the integrals $I_{s}(a), s=0,1,2$ gives:

$$
I_{s}(a) \cong \rho \frac{\xi_{0}}{d} \exp \left(-\frac{d}{\xi_{0}}\right), \quad \lambda \gg 1 .
$$

and, thus, currently we obtain the expression

$$
j \cong 2,5 \frac{e n v_{0}}{p_{0} d} \mathrm{e}^{-d / \xi_{0}}\left(1-\frac{T}{T_{\mathrm{c}}}\right)^{2} \sin \varphi, d \gg \xi_{0} .
$$

\section{The common equation for NIS-junction with nonmagnetic impurities}

In a common case, when the reflecting of electrons from an interface is available, a set of linear integral equations, written in the dimensionless quantities, has the 
form $[1, \S 40]$ :

$$
\begin{aligned}
\Delta(\zeta)=\frac{\rho}{2} \theta(\zeta) \int_{-\infty}^{\infty} \mathrm{d} \zeta^{\prime} \sum_{n} \int_{0}^{1} \frac{\mathrm{d} x}{x}\left\{\exp \left(-\frac{\left|\zeta-\zeta^{\prime}\right|}{x}\left|2 n^{\prime}+1\right|\right)\right. \\
\left.+R(x) \operatorname{sign} \zeta \zeta^{\prime} \exp \left(-\frac{|\zeta|+\left|\zeta^{\prime}\right|}{x}\left|2 n^{\prime}+1\right|\right)\right\} \Delta_{n}\left(\zeta^{\prime}\right) \\
\Delta_{n}(\zeta)=\Delta(\zeta)+\frac{1}{2 \lambda} \int_{-\infty}^{\infty} \mathrm{d} \zeta^{\prime} \int_{0}^{1} \frac{\mathrm{d} x}{x}\left\{\exp \left(-\frac{\left|\zeta-\zeta^{\prime}\right|}{x}\left|2 n^{\prime}+1\right|\right)\right. \\
\left.+R(x) \operatorname{sign} \zeta \zeta^{\prime} \exp \left(-\frac{|\zeta|+\left|\zeta^{\prime}\right|}{x}\left|2 n^{\prime}+1\right|\right)\right\} \Delta_{n}\left(\zeta^{\prime}\right)
\end{aligned}
$$

Although now one cannot exclude $\Delta_{n}(\zeta)$ and obtain a closed equation for $\Delta(\zeta)$, the asymptotical properties of the equation solutions in $+\infty$ remain. Really, if $\zeta \rightarrow \infty$, then the additional terms, associated with $R$, disappear from the equation kernels, and the asymptotical form of both equations does not contain $R$ any more. If we denote $\zeta-\zeta^{\prime}=u$, and take into account that in the integrand $\zeta \gg u$, we can write

$$
\Delta_{n}(\zeta) \stackrel{\text { as }}{=} \Delta(\zeta)+\frac{1}{2 \lambda} \int_{-\infty}^{\infty} \mathrm{d} u\left(\Delta_{n}(\zeta)+\frac{1}{2} \Delta_{n}^{\prime \prime}(\zeta) u^{2}\right) \int_{0}^{1} \frac{\mathrm{d} x}{x} \exp \left(-\frac{|u|}{x}\left|2 n^{\prime}+1\right|\right)
$$

i.e.

$$
\Delta_{n}(\zeta) \stackrel{\text { as }}{=} \Delta(\zeta)+\frac{1}{\lambda} \frac{\Delta_{n}(\zeta)}{\left|2 n^{\prime}+1\right|}+\frac{1}{3 \lambda} \frac{\Delta_{n}^{\prime \prime}(\zeta)}{\left|2 n^{\prime}+1\right|^{3}}
$$

The first equation will have the asymptotical form

$$
\Delta(\zeta) \stackrel{\text { as }}{=} \rho \sum_{n} \frac{\Delta_{n}(\zeta)}{\left|2 n^{\prime}+1\right|}+\frac{\rho}{3} \sum_{n} \frac{\Delta_{n}^{\prime \prime}(\zeta)}{\left|2 n^{\prime}+1\right|^{3}} .
$$

Expressing $\Delta_{n}(\zeta)$ from equation (27) and substituting it to equation (28) we obtain

$$
\Delta(\zeta)=\rho \sum_{n} \frac{1}{|2 n+1|} \Delta(\zeta)+\frac{\rho}{3 \lambda} \sum_{n} \frac{\Delta_{n}^{\prime \prime}(\zeta)}{|2 n+1|\left|2 n^{\prime}+1\right|^{3}}+\frac{\rho}{3} \sum_{n} \frac{\Delta_{n}^{\prime \prime}(\zeta)}{\left|2 n^{\prime}+1\right|^{3}} .
$$

$\Delta(\zeta)$ drops out from this equation and the terms with the second derivative are reduced. As a result

$$
\frac{\rho}{3} \sum_{n} \frac{\Delta_{n}^{\prime \prime}(\zeta)}{|2 n+1|\left|2 n^{\prime}+1\right|^{3}}=0
$$

from where $\Delta^{\prime \prime}=0$. And taking into account (27)

$$
\Delta_{n}(\zeta) \stackrel{\text { as }}{=}\left|\frac{2 n^{\prime}+1}{2 n+1}\right| \Delta(\zeta)
$$


From the asymptotical equality of the derivatives $\Delta^{\prime \prime}(\zeta)$ and $\Delta_{n}^{\prime \prime}(\zeta)$ to zero one can conclude, that the asymptotics of the solutions of the integral equation on $+\infty$ are linear:

$$
\begin{aligned}
\Delta(\zeta) & \stackrel{\text { as }}{=} \zeta+q_{\infty}, \\
\Delta_{n}(\zeta) & =\left|\frac{2 n^{\prime}+1}{2 n+1}\right|\left(\zeta+q_{\infty}\right), \quad \zeta \rightarrow+\infty
\end{aligned}
$$

Obviously, at $\zeta \rightarrow-\infty$ asymptotics is zero, because $\Delta(\zeta)=0$ at $\zeta<0$, and $\Delta_{n}(\zeta)$ goes to zero if $\zeta \rightarrow-\infty$, as one can see from the equation. The asymptotics is extracted as usually, and here we shall write it in the form $A \zeta+B$, that gives more possibilities afterwards, for example, we can include a case $A=0 \quad\left(q_{\infty}=\infty\right)$. So we write

$$
\begin{aligned}
\Delta(\zeta) & =(A \zeta+B) \theta(\zeta)+\psi(\zeta), \\
\Delta_{n}(\zeta) & =\left|\frac{2 n^{\prime}+1}{2 n+1}\right|(A \zeta+B) \theta(\zeta)+\psi_{n}(\zeta) .
\end{aligned}
$$

After extracting the asymptotics, the terms that do not go to zero on the infinity are cancelled and the equations get homogeneous.

$$
\begin{aligned}
& \psi_{n}(\zeta)-\psi(\zeta)-\frac{1}{2 \lambda} \int_{-\infty}^{\infty} \mathrm{d} \zeta^{\prime} \psi_{n}\left(\zeta^{\prime}\right) \int_{0}^{1} \frac{\mathrm{d} x}{x} \\
& \times\left\{\exp \left(-\frac{\left|\zeta-\zeta^{\prime}\right|}{x}\left|2 n^{\prime}+1\right|\right)+\mathcal{R}(x) \operatorname{sign} \zeta \zeta^{\prime} \exp \left(-\frac{|\zeta|+\left|\zeta^{\prime}\right|}{x}\left|2 n^{\prime}+1\right|\right)\right\}= \\
& =\frac{A}{2 \lambda|2 n+1|\left|2 n^{\prime}+1\right|} \int_{0}^{1} \mathrm{~d} x x(1+\mathcal{R}(x) \operatorname{sign} \zeta) \exp \left(-\frac{\left|2 n^{\prime}+1\right|}{x}|\zeta|\right) \\
& \quad-\frac{B \operatorname{sign} \zeta}{2 \lambda|2 n+1|} \int_{0}^{1} \mathrm{~d} x \mathcal{D}(x) \exp \left(-\frac{\left|2 n^{\prime}+1\right|}{x}|\zeta|\right) \cdot \\
& \psi(\zeta)-\frac{\rho}{2} \theta(\zeta) \int_{-\infty}^{\infty} \mathrm{d} \zeta^{\prime} \sum_{n} \psi_{n}\left(\zeta^{\prime}\right) \int_{0}^{1} \frac{\mathrm{d} x}{x} \\
& \times\left\{\exp \left(-\frac{\left|\zeta-\zeta^{\prime}\right|}{x}\left|2 n^{\prime}+1\right|\right)+\mathcal{R}(x) \operatorname{sign} \zeta \zeta^{\prime} \exp \left(-\frac{|\zeta|+\left|\zeta^{\prime}\right|}{x}\left|2 n^{\prime}+1\right|\right)\right\}= \\
& =A \frac{\rho}{2} \theta(\zeta) \sum_{n} \frac{1}{|2 n+1|\left|2 n^{\prime}+1\right|} \int_{0}^{1} \mathrm{~d} x x(1+\mathcal{R}(x)) \exp \left(-\frac{\left|2 n^{\prime}+1\right|}{x} \zeta\right) \\
& \quad-B \frac{\rho}{2} \theta(\zeta) \sum_{n} \frac{1}{|2 n+1|} \int_{0}^{1} \mathrm{~d} x \mathcal{D}(x) \exp \left(-\frac{\left|2 n^{\prime}+1\right|}{x} \zeta\right)
\end{aligned}
$$

We shall give here a form of the variation method that can be called a method of quasiorthogonality to asymptotics. Let us integrate both equations by $\zeta$ from zero 
to infinity. We obtain

$$
\begin{aligned}
& \int_{0}^{\infty} \psi_{n}(\zeta) \mathrm{d} \zeta=\frac{\left|2 n^{\prime}+1\right|}{|2 n+1|} \int_{0}^{\infty} \psi(\zeta) \mathrm{d} \zeta \\
& \quad-\frac{1}{2 \lambda|2 n+1|} \int_{-\infty}^{\infty} \mathrm{d} \zeta \psi_{n}(\zeta) \operatorname{sign} \zeta \int_{0}^{1} \mathrm{~d} x \mathcal{D}(x) \exp \left(-\frac{\left|2 n^{\prime}+1\right|}{x}|\zeta|\right) \\
& \quad+\frac{A}{2 \lambda\left|2 n^{\prime}+1\right|\left(2 n^{\prime}+1\right)^{2}} \int_{0}^{\infty} \mathrm{d} x x^{2}(1+\mathcal{R}(x))-\frac{B}{2 \lambda(2 n+1)^{2}} \int_{0}^{1} \mathrm{~d} x x \mathcal{D}(x), \\
& \int_{0}^{\infty} \psi(\zeta) \mathrm{d} \zeta-\rho \sum_{n} \frac{1}{\left|2 n^{\prime}+1\right|} \int_{0}^{\infty} \psi_{n}(\zeta) \mathrm{d} \zeta \\
& \quad+\frac{\rho}{2} \sum_{n} \frac{1}{\left|2 n^{\prime}+1\right|} \int_{-\infty}^{\infty} \mathrm{d} \zeta \psi_{n}(\zeta) \operatorname{sign} \zeta \int_{0}^{1} \mathrm{~d} x \mathcal{D}(x) \exp \left(-\frac{\left|2 n^{\prime}+1\right|}{x}|\zeta|\right)= \\
& \quad=A \frac{\rho}{2} \sum_{n} \frac{1}{|2 n+1|\left(2 n^{\prime}+1\right)^{2}} \int_{0}^{1} x^{2}(1+\mathcal{R}(x)) \mathrm{d} x \\
& \quad-B \frac{\rho}{2} \sum_{n} \frac{1}{|2 n+1|\left|2 n^{\prime}+1\right|} \int_{0}^{1} x \mathcal{D}(x) \mathrm{d} x .
\end{aligned}
$$

Now let us substitute the expression (33) into equation (34). After simplification we have an equality

$$
\begin{aligned}
& \sum_{n} \frac{1}{|2 n+1|} \int_{-\infty}^{\infty} \mathrm{d} \zeta \psi_{n}(\zeta) \operatorname{sign} \zeta \int_{0}^{1} \mathrm{~d} x \mathcal{D}(x) \exp \left(-\frac{\left|2 n^{\prime}+1\right|}{x}|\zeta|\right)= \\
& =A \sum_{n} \frac{1}{\left|2 n^{\prime}+1\right|(2 n+1)^{2}} \int_{0}^{1} x^{2}(1+\mathcal{R}(x)) \mathrm{d} x-B \sum_{n} \frac{1}{(2 n+1)^{2}} \int_{0}^{1} x \mathcal{D}(x) \mathrm{d} x .
\end{aligned}
$$

Similar manipulations should be performed with equations (31), (32), multiplied by $\zeta$ and integrated by $\zeta$. Thus, in the same way we substitute an expression for $\int_{0}^{\infty} \psi_{n}(\zeta) \zeta \mathrm{d} \zeta$ into the relation for $\int_{0}^{\infty} \psi(\zeta) \zeta \mathrm{d} \zeta$ and after all simplifications we obtain

$$
\begin{aligned}
\sum_{n} & \frac{1}{|2 n+1|\left|2 n^{\prime}+1\right|} \int_{-\infty}^{\infty} \mathrm{d} \zeta \psi_{n}(\zeta) \int_{0}^{1} \mathrm{~d} x x(1+\mathcal{R}(x) \operatorname{sign} \zeta) \exp \left(-\frac{\left|2 n^{\prime}+1\right|}{x}|\zeta|\right)= \\
= & B \sum_{n} \frac{1}{(2 n+1)^{2}\left|2 n^{\prime}+1\right|} \int_{0}^{1} x^{2} \mathcal{D}(x) \mathrm{d} x \\
& -A \sum_{n} \frac{1}{(2 n+1)^{2}\left(2 n^{\prime}+1\right)^{2}} \int_{0}^{1} x^{3}(1+\mathcal{R}(x)) \mathrm{d} x
\end{aligned}
$$

We shall show how to use the method of quasiorthogonality for the approximate calculation of the coefficient $q_{\infty}$ in case when on the NS interface we have a reflection 
and the dimensionless free path length $\lambda \gg 1$. Let us rewrite equations (35) and (36) in the form of a relation that contains a function $q_{n}(\zeta)=\psi_{n}(\zeta)+\mid\left(2 n^{\prime}+1\right) /(2 n+$ 1) $\mid q_{\infty} \theta(\zeta)$. Here we introduce the notation

$$
\chi\left(\lambda^{-1}\right)=\sum_{n} \frac{1}{(2 n+1)^{2}\left|2 n^{\prime}+1\right|}, \quad \chi_{1}\left(\lambda^{-1}\right)=\sum_{n} \frac{1}{(2 n+1)^{2}\left(2 n^{\prime}+1\right)^{2}} .
$$

This way, instead of equations (35) and (36) we obtain the following:

$$
\begin{aligned}
& \sum_{n} \frac{1}{|2 n+1|} \int_{-\infty}^{\infty} \mathrm{d} \zeta q_{n}(\zeta) \operatorname{sign} \zeta \int_{0}^{1} \mathrm{~d} x \mathcal{D}(x) \exp \left(-\frac{\left|2 n^{\prime}+1\right|}{x}|\zeta|\right)= \\
& \quad=\chi\left(\lambda^{-1}\right) \int_{0}^{1}(1+\mathcal{R}(x)) x^{2} \mathrm{~d} x \\
& \sum_{n} \frac{1}{|2 n+1|\left|2 n^{\prime}+1\right|} \int_{-\infty}^{\infty} \mathrm{d} \zeta q_{n}(\zeta) \int_{0}^{1} \mathrm{~d} x x(1+\mathcal{R}(x) \operatorname{sign} \zeta) \exp \left(-\frac{\left|2 n^{\prime}+1\right|}{x}|\zeta|\right)= \\
& \quad=\frac{2}{3} q_{\infty} \chi\left(\lambda^{-1}\right)-\chi_{1}\left(\lambda^{-1}\right) \int_{0}^{1} x^{3}(1+\mathcal{R}(x)) \mathrm{d} x
\end{aligned}
$$

Writing a trial function in the form $q_{n}(\zeta) \equiv \theta(\zeta)\left|\left(2 n^{\prime}+1\right) /(2 n+1)\right| \Gamma$, where $\Gamma=$ const, we get from the relation (37) and (38) the following:

$$
q_{\infty}=\frac{3}{2} \frac{\chi_{1}\left(\lambda^{-1}\right)}{\chi\left(\lambda^{-1}\right)} \int_{0}^{1} x^{2}(1+\mathcal{R}(x)) \mathrm{d} x+\frac{3}{2} \frac{\chi\left(\lambda^{-1}\right)\left[\int_{0}^{1} x^{2}(1+\mathcal{R}(x)) \mathrm{d} x\right]^{2}}{S_{2} \int_{0}^{1} x \mathcal{D}(x) \mathrm{d} x} .
$$

This formula contains a result for NIS surface at the presence of impurities, including $\left(l \gg \xi_{0}\right)$. But the formula

$$
q_{\infty} \stackrel{\text { as }}{=} \frac{2}{3} \frac{\chi\left(\lambda^{-1}\right)}{S_{2}}\left(\int_{0}^{1} x \mathcal{D}(x) \mathrm{d} x\right)^{-1}
$$

is asymptotically exact at small $\mathcal{D}$ for arbitrary concentrations of impurities, including $l \ll \xi_{0}$, when it gets the form

$$
q_{\infty} \stackrel{\text { as }}{=} \frac{2}{3} \lambda\left(\int_{0}^{1} x \mathcal{D}(x) \mathrm{d} x\right)^{-1}
$$

To obtain this solution, we have to note, that when $\mathcal{R}=1$ the set of equations (25), (26) is satisfied by the constant $\Delta_{n}=\left|\left(2 n^{\prime}+1\right) /(2 n+1)\right| \Delta, \Delta=$ const. It means, that in this case $A=0$ and $B$ is any constant that can be taken as a unit.

If $\mathcal{D}$ is small, one can apply the perturbation theory by $\mathcal{D}$, taking for zero approximation $A^{(0)}=0, B^{(0)}=1$ and $\psi_{n}^{(0)}(\zeta)=0, \psi^{(0)}(\zeta)=0$. Considering now (35) we see that the left part of this relation is a quantity of the second order by 
$D$ and thus can be neglected in the first approximation. Then equation (35) gives $A^{(1)} \frac{2}{3} \chi\left(\lambda^{-1}\right)=S_{2} \int_{0}^{1} x \mathcal{D}(x) \mathrm{d} x$, because $\mathrm{B}$ is equal to unit in the zero approximation. Thus, for $q_{\infty}=1 / A$ we obtain (39). In particular, for $\lambda \ll 1$ in the expression for $\chi\left(\lambda^{-1}\right)$ we can let

$$
\chi\left(\lambda^{-1}\right)=\sum_{n} \frac{1}{(2 n+1)^{2}\left|2 n^{\prime}+1\right|}=\sum_{n} \frac{1}{(2 n+1)^{2}\left[\left|2 n^{\prime}+1\right|+1 / \lambda\right]} \cong \lambda S_{2} .
$$

Thus we obtain an asymptotically exact expression (40).

\title{
References
}

1. Svidzinsky A.V. Space-Inhomogeneous Problems of the Superconducting Theory. Moscow, Nauka, 1982.

2. Svidzinsky A.V. The theory of the Josephson's currents in superconducting junctions with normal layer. - In: Problems of the Modern Statistical Physics. Kyiv, Naukova dumka, 1985, p. 245-256.

\section{Струмові стани в SNS-контакті за наявності немагнітних домішок довільної концентрації}

\author{
А.В.Свідзинський, В.Є.Сахнюк \\ Кафедра теоретичної і математичної фізики, \\ Волинський державний університет ім. Лесі Українки, \\ 43000 Луцьк, проспект Волі, 13 \\ Отримано 18 квітня 2000 р.
}

Отримано граничні умови на NS-границі за наявності немагнітних домішок довільної концентрації. Розглянуто струмові стани в SNSконтакті. Зроблено аналіз отриманого виразу для струму, зокрема з'ясовано, як відбувається перехід від характерної довжини спадання струму $\xi_{0}$ для чистого надпровідника до $\xi_{\mathrm{d}}$ у випадку забрудненого. Для отримання граничних умов використаний варіант варіаційного методу, названий методом квазіортогональності до асимптотики.

Ключові слова: надпровідник, параметр впорядкування, домішки, функція Гріна

PACS: $74.50 .+r$ 